\title{
APARICIÓN DE STAPHYLOCOCCUS AUREUS EN LOS HOSPITALES: UNA REVISIÓN DE LITERATURE
}

RODRIGUES, Sheila da Conceição Sousa ${ }^{1}$

Fecury, Amanda Alves ${ }^{2}$

Dias, Cláudio Alberto Gellis De Mattos ${ }^{3}$

OLIVEIRA, Euzébio de ${ }^{4}$

RODRIGUES; et. al. - Aparición de Staphylococcus Aureus en los hospitales: un literatura Informe - Multidisciplinario base conocimiento científico revista Vol. 2. Año. 1. Mayo. 2016, pp. 33-42 - ISSN: 0959-2448

\section{RESUMEN}

El presente estudio intentó compilar datos sobre la resistencia a los antimicrobianos de las bacterias del género Staphylococcus aureus en hospitales públicos brasileños, años 2008-2015. Los datos encontrados en los artículos publican en revistas nacionales e internacionales, a través de búsqueda activa en el período 2014-2015,

${ }^{1}$ Biólogo. Especialista en Microbiología de la Universidad Ipiranga, PA. Correo electrónico: microbiologistasheilarodrigues12@hotmail.com

2 Biomédica. Doctorado en enfermedades tropicales. Investigador en el programa graduado del centro de Medicina Tropical de la Facultad y la Universidad investigadora UFPA Federal do Amapá, AP. Correo electrónico: amanda_fecury@yahoo.com.br ${ }^{3}$ Biólogo. Doctor en teoría e investigación. Profesor e investigador en el Instituto del estado de Amapá-FIPA Federal. Correo electrónico: claudiodias66@gmail.com 4 Eusebio. Biólogo. Doctor en Medicina Tropical enfermedades. Investigador del programa graduado del centro de Medicina Tropical de la UFPA. Profesor e investigador de la Universidad Federal de Pará - UFPA. Correo electrónico: euzebio21@yahoo.com.br (autor corresponsal). 
con palabras clave: Staphylococcus aureus en hospitales, lesiones causadas por Staphylococcus aureus en hospitales públicos brasileños y resistencia de Staphylococcus aureus a los antimicrobianos. Los resultados mostraron una gran prevalencia de unidades de meticilina en el hospital de aureus MRSA de estafilococo multidrug-resistentes en Brasil, según los artículos analizados, publicados entre los años 2008 y 2015. Se concluye que el estafilococo son patógenos que quedan en el ambiente del hospital, creando cierta resistencia a determinados medicamentos, que ha contribuido a una mayor investigación con respecto al uso indiscriminado de antimicrobianos en la comunidad y medicamentos más eficaces para el tratamiento de estos microorganismos en unidades hospitalarias.

Palabras clave: Staphylococcus aureus, unidad hospitalaria, resistencia a los antimicrobianos.

\section{INTRODUCCIÓN}

La presencia de microorganismos tales como bacterias del género Staphylococcus aureus en unidades hospitalarias en los últimos años ha crecido considerablemente, debido a la resistencia de estos microorganismos para ciertos tipos de antimicrobianos, que ha causado importantes patologías. Sí, las bacterias s. aureus están responsables de varias lesiones en el individuo.

Las lesiones que este tipo de bacterias puede ser causa solamente superficial como la piel (epidermitis) o del tipo invasivo como endocarditis, osteomielitis, artritis, miositis y neumonía, además el síndrome de piel escaldada, y alimentos intoxicación por dependiendo de la etapa bacteriana, pueden llevar a la muerte. (más invasivos), que depende de su estructura, generalmente de un solo gen de los cromosomas bacterianos o plásmidos ${ }^{12}$.

Los cuadros clínicos suelen ser comunes en pacientes sometidos a unidades hospitalarias, como el s. aureus, un microorganismo común en estos ambientes. Además de permanecer durante un largo periodo de tiempo, adheridos a objetos 
inanimados como en el hospital o muebles en pacientes asintomáticos que pueden infectar a la comunidad en General ${ }^{3}$.

Los estudios demuestran que Staphylococcus son microorganismos patológicos antiguos humanos, descritos por Robert Koch en 1878 y cultivadas por Louis Pasteur en 1880, los microorganismos pertenecen a la familia de coccus gram positivos llamados micrococcaceae y s. aureus el principal patógeno humano. Producen enzimas coagulaze, producen pus y toxinas, también puede conducir a bacteriemia y endocarditis por el torrente sanguíneo, presenta mecanismos patogénicos como la destrucción de tejido que es la aparición del absceso en la piel, articulaciones, cerebro y pulmones, así como el síndrome de piel escaldada, choque tóxico y el envenenamiento de alimentos ${ }^{1-4}$.

El s. aureus se ha vuelto cada vez más presente en las unidades del hospital por ser un microorganismo que con los años ha demostrado cepas cada vez más resistentes a ciertos tipos de antibióticos. Por lo tanto, cuanto mayor sea la cantidad de droga para el tratamiento de infecciones causadas por bacterias, mayor será la probabilidad de encontrar resistentes a cepas como aureus $5 \mathrm{~s}$. SARM resistente a la meticilina.

Debido a este problema hubo la necesidad de reconocer las principales causas de la presencia de s. aureus en los hospitales públicos brasileños, como una de las principales causas de daños, haciendo hincapié en la fuerza de estos microorganismos para ciertos tipos de antibióticos, que se describen en el curso de esta investigación.

La presencia de bacterias del género de s. aureus en unidades hospitalarias, se ha estudiado con frecuencia en los últimos años por investigadores de todo el mundo, como resultado de cepas resistentes a los antibióticos, que tuvo como objetivo la realización de esta investigación, en busca de datos publicados en los artículos informe la resistencia de s. aureus a los antimicrobianos utilizados en los hospitales públicos, para el tratamiento de las patologías presentadas por esta bacteria adquirida en la comunidad o en el entorno de hospital propio, de los cuales fueron descritos anteriormente. 


\section{MÉTODO}

Se refiere a un estudio retrospectivo realizado en periodo de 2014 a 2015, con encuesta en bases de datos, con el fin de coleccionismo de artículos científicos publicados en revistas nacionales e internacionales desde 2008 hasta 2015, en la aparición de cepas de bacterias del género $s$. aureus resistentes a los antimicrobianos en los hospitales públicos de Brasil.

UNI-términos fueron utilizados: Staphylococcus aureus, unidad hospitalaria, resistencia a los antimicrobianos. 60 Articulos relacionados a la investigación fueron seleccionados y analizados, de los cuales quince fueron elegidos para guiar la investigación, incluyendo sólo aquellos que tienen mayor relevancia. Confirmando que el artículo sexto último estudiado, es un estudio de caso, con patologías graves en un niño de siete años.

Este método permitida encontrar distintos artículos relacionados con la investigación sobre la presencia de $\mathrm{s}$. aureus en los hospitales públicos brasileños de los cuales se presentan a continuación.

\section{RESULTADOS}

Tras el análisis de quince artículos más relevantes para buscar seis se destacaron, mostrando resultados que caracterizan la búsqueda relativa a la presencia de $\mathrm{s}$. aureus en los hospitales.

El artículo de Vieira y Agostini, 2008 destacó la prevalencia y el perfil de infecciones causadas por s. aureus en un hospital público. El artículo de Cruvinel, Silveira et al., 2011 había destacado perfil antimicrobiano de s. aureus en una UCl en el Distrito Federal. Artículo de Moura, pimienta et al..2011 retrató la colonización de profesionales de la salud de un hospital de Sao Paulo para s. aureus. Artículo de Castro, Barth et al..2009 determinó la resistencia antimicrobiana de s. aureus se aísla de un laboratorio de hospital. Artículo de Souza y Figueiredo, 2008 puso de relieve la prevalencia de las infecciones nosocomiales causadas por s. aureus en un hospital 
regional. Y el artículo de Gelatti,.2009 Sukiennik reportaron un estudio de caso con el paciente de 7 años de edad, residente en Porto Alegre que presenta residuos sépticos causada por s. aureus, permaneció hospitalizado durante 50 días.

Se discutirán los artículos debajo de nombre de donde los autores llevan a cabo la investigación. Artículo de oro informa s. aureus como un microorganismo importante el ambiente de hospital que sobrevive en objetos inanimados o en pacientes asintomáticos y dirigida a buscar nueva información sobre el perfil de infecciones causadas por este microorganismo y los factores que contribuyeron a su aislamiento en el hospital. Se llevó a cabo el método utilizado por los autores de 64 registros de pacientes ingresados por infecciones de s. aureus en el período de enero a diciembre de 2008, en el que los datos se recolectaron entre junio y julio de 2009. De un total de 617 cultivo placas se encontraron 64 resultados positivos para s. aureus, es decir, el $10.37 \%$ del total. Lo que puede confirmar la presencia de este microorganismo en este hospital unidad ${ }^{3}$.

Distrito Federal caracteriza s. aureus como el principal agente causal de la infección, siendo el principal lugar de colonización nasal de estos microorganismos en individuos sintomáticos o asintomáticos, así como su resistencia a MRSA (resistente a la meticilina) en la escala global y otros antimicrobianos, como vancomicina por ejemplo. Los métodos de muestreo nasal autores utilizadas recogen con hisopo de pacientes de ICU 20 (reciente hospitalización menos de una semana) en el período de febrero a mayo de 2010.

Los resultados para estos aislamientos de s. aureus $07-37 \%$ fueron resistentes a amoxicilina, $12-60 \%$ eran resistentes a ampicilina y solamente $3-15 \%$ resistente a la ampicilin ${ }^{\mathrm{a}} 6$.

Artículo de São Paulo retrata la presencia de s. aureus en la saliva de enfermería personal de un hospital de enseñanza, con miras a determinar la sensibilidad antimicrobiana y también la caracterización fenotípica de s. aureus. El método utilizado fue un estudio epidemiológico transversal, realizado en el período de enero a diciembre de 2007, en las unidades de cuidados intensivos (UCl) de un hospital 
público grande en el país. Los sujetos de la investigación fueron 351 enfermeras, técnicos y auxiliares de enfermería en la actividad profesional, de los cuales tres métodos: se tomaron muestras de cada tema, con dos meses de cada colección, un total de 1.053. Estas muestras han computado en un resultado de 17.6 (126) del total positivo para s. aureus espectáculos. Y, cuando la identificación de la sensibilidad antimicrobiana, de este total, 26 metricilina MRSA resistente, siendo la otra como sensivel7 MSS $^{\mathrm{A}}$.

Artículo de Santa María pone en evidencia el s. aureus como un problema emergente que trae preocupación a los hospitales como comunidades. Observa también que la mortalidad asociada a s. aureus ha disminuido con el uso de penicilina y cepas resistentes han surgido últimamente esa mierda (resistente a la meticilina Staphylococcus aureus-MRSA). El método del investigación, 34 aislamientos de s. aureus previamente caracterizado como fenotípicamente resistentes a la oxacilina, obtenida de una de hospital Santa Maria/RS. Los resultados de la informática en la sensibilidad de los aislados de s. aureus a la vancomicina antimicrobiana, que hizo evidente la mayor efectividad de la droga para tratar a pacientes colonizados por ese tipo de microorganismo, frente de trece fármacos diferentes, incluyendo ciprofloxacina8 y azitromicina de gentamicina.

El artículo de Paraná se refiere a los cambios que han ocurrido en los años en relación con la medicina y su demanda de antibióticos más eficaces, con el fin de caracterizar la prevalencia de mosocomiais infecciones causadas por s. aureus MRSA, un hospital regional de Maringá. El método de recolección de datos fue mediante un estudio retrospectivo basado en datos de control de infecciones hospital Comité. Los resultados obtenidos entre febrero de 2003 y diciembre de 2006 de un total de 68 casos de s. aureus MRSA, $16.2 \%$ de esta cantidad para la infección de MRSA en el año de 2003, con un incremento de $62.2 \%$ en $200^{5} 9$.

La comunidad del sur de Brasil divulga un caso de paciente masculino de 7 años de edad, residente de la región metropolitana de Porto Alegre, que presentó un marco de sepsis grave por s. aureus, neumonía grave secundaria a lesión suave del tejido en la izquierda miembro inferior evolucionado para celulitis que en las radiografías, 
afectación ósea, presencia de abscesos hepáticos, abscesos de pulmón y varios hemoptise ${ }^{10-16}$.

El caso muestra cómo investigación en s. aureus en los hospitales y en la comunidad en general, buscando un mayor conocimiento con respecto al tratamiento a través de los antimicrobianos, con su forma de resistencia en ciertos casos y ciertas cepas, haciéndonos creer que es cada vez más difícil de contener enfermedades que sólo con los agentes antimicrobianos que se han desarrollado hasta ahora. Ese legado requiere más profundo para aclarar mejores los estudios subsecuentes por la gran presencia de s. aureus en los hospitales y en la comunidad, hacer su tratamiento más eficaz frente a estas enfermedades graves como la implicación del paciente.

Presentan artículos que guió la investigación posibles determinantes de los resultados de antimicrobianos en el tratamiento de s. aureus como la edad, enfermedad subyacente, tiempo de hospitalización y otros que serán discutidos en la tabla 1, abajo:

\begin{tabular}{|l|l|}
\hline ARTÍCULOS & DETERMINANTES \\
\hline DOURADOS & Grupo de edad \\
\hline Sexo & Duración de la estancia comorbilidades \\
& Enfermedad subyacente \\
& Dispositivo invasivo \\
& Resistencia del microorganismo \\
\hline DISTRITO FEDERAL & $\begin{array}{l}\text { No establecido } \\
\text { Sexo }\end{array}$ \\
\hline SÃO PAULO & Edad \\
\hline
\end{tabular}




\begin{tabular}{|c|c|}
\hline & CAMBIO y viaje al trabajo \\
\hline SANTA MARIA & No establecido \\
\hline MARINGA & Duración de la estancia y edad \\
\hline PORTO ALEGRE & $\begin{array}{l}\text { Hospitalización en unidad de cuidados } \\
\text { intensivos } \\
\text { Hospitalización prolongada } \\
\text { Enfermedad subyacente grave } \\
\text { Procedimientos invasivos } \\
\text { Uso prolongado de antibióticos }\end{array}$ \\
\hline
\end{tabular}

Tabla 01. Posibles determinantes de la resistencia antimicrobiana de s. aureus

Fuente: datos encontrados en los artículos estudiados.

Estos posibles determinantes son de primordial importancia para explicar aún más determinadas patologías presentadas por $\mathrm{s}$. aureus, siendo que una historia del paciente siempre debe ser claro. $Y$ los antimicrobianos cuyas pruebas fueron realizadas en pacientes y señalaron artículos sobre resistencia o sensibilidad de ciertas cepas de $\mathrm{s}$. aureus deben tenerse en cuenta en la búsqueda de respuestas satisfactorias para el tratamiento de esta bacteria en unidades hospitalarias.

La tabla 2, entonces algunos de los antimicrobianos específicos probados en laboratorios según datos de la investigación:

\begin{tabular}{|l|l|}
\hline RESISTENCIA & SENSIBILIDAD \\
\hline \multicolumn{1}{|c|}{ DOURADOS } & \\
\hline & \\
\hline Penicilina & Vancomicina \\
\hline
\end{tabular}




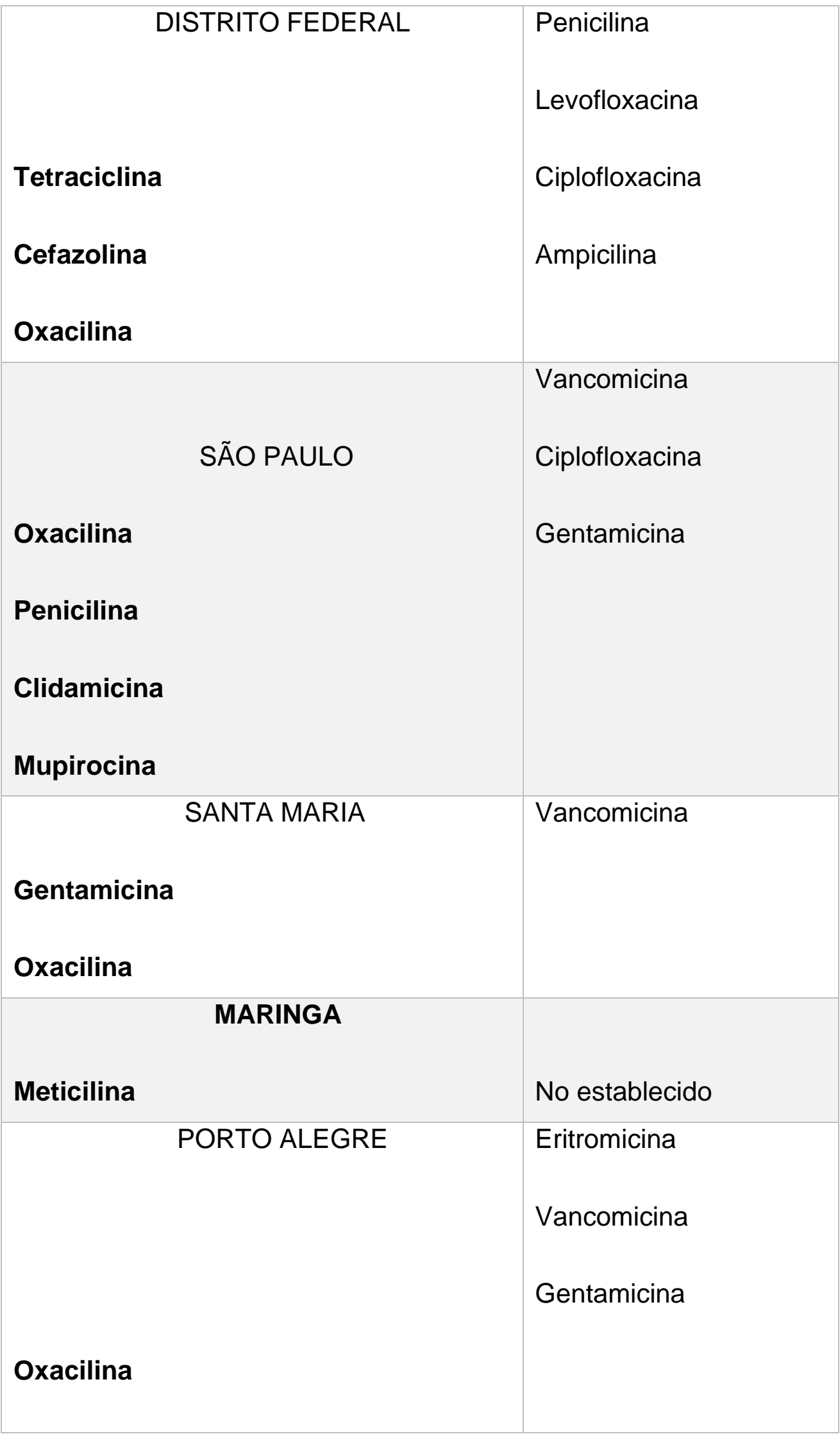




\section{Cefoxitina}

Tabla 02. Más usado como resistencia o susceptibilidad de s. aureus.

Fuente: datos encontrados en los artículos estudiados.

Probar datos con la mayoría de las pruebas llevadas a cabo para identificar el perfil de resistencia o sensibilidad de las bacterias $\mathrm{s}$. aureus conformidad con los artículos estudiados es que en la mayoría de estas pruebas vancomicina ${ }^{11}$ es señalado como el antimicrobiano más efectivo para el tratamiento de patologías presentadas por estas bacterias, lo que es sensible, siendo el grupo de los que todavía tienen una mayor resistencia a los antimicrobianos verdadera con respecto a s. aureus. Lo que justifica la presencia de estas bacterias en unidades hospitalarias.

\section{CONCLUSIÓN}

La revisión de la literatura en el contexto científico es de vital importancia en futuras investigaciones en varias áreas del conocimiento, porque proporcionan datos concretos y documentos relacionados con estos asuntos, lo que es un factor relevante en la búsqueda de nuevos temas en este largo proceso. Y, mientras investigamos la presencia de $\mathrm{s}$. aureus en unidades hospitalarias incluso a través de esta investigación bibliográfica, nos dimos cuenta que la principal preocupación con respecto a la presencia de estas bacterias es con su fuerza, entendiendo que estos microorganismos pueden sobrevivir en objetos inanimados, así como en pacientes asintomáticos que adquieran este microorganismo en la comunidad de llevarlos al hospital, ahora en el entorno hospitalario como era habitual que puede contaminar a otros pacientes por ser un patógeno común en micro biota del tabique nasal y en micro biota de la piel.

Y hoy en día, la presencia repentina de estos microorganismos o simples en los hospitales, especialmente en público donde la mayoría de la población, ha sido la manzana de los ojos de muchos investigadores que tienen que estudiar y hablar de drogas cada vez más eficaz en el tratamiento de pacientes colonizados por estos 
microorganismos, dejando la resistencia de cepas de SARM con sensibilidad MSSA y pueden existir encontrado VRSA resistente-Staphylococcus aureus resistentes a la vancomicina, hasta entonces dicta cómo el fármaco más eficaz en el tratamiento de esta bacteria, que permite nuevos estudios a prueba de dicha evolución de cepas serían resistente a la vancomicina.

Y esta es la causa de mayor preocupación de los profesionales de la salud, así como el uso indiscriminado de antibióticos en la comunidad, lo que agrava aún más los problemas de $\mathrm{s}$. aureus en unidades hospitalarias, así como otros microorganismos que necesitan ser tratados con antimicrobianos.

Se dan cuenta que este es un tema que merece una mirada más considerada de todos los que se ocupan de salud y todo lo que necesita ser más saludables, por lo que pueden la policía frente a la presencia de $\mathrm{s}$. aureus de la comunidad o en unidades hospitalarias, ya que su principal reservorio es el hombre que puede o no puede mostrar síntomas de esta bacteria. $Y$ de esta manera, la presencia de $\mathrm{s}$. aureus en los hospitales públicos brasileños podría ser menos constante que ha sido hasta ahora.

\section{REFERENCIAS}

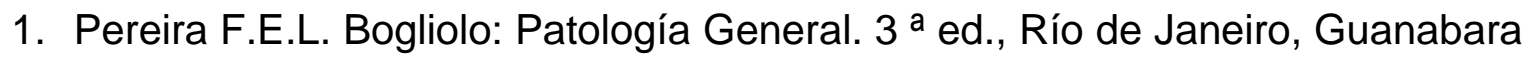
Koogam, 2004.

2. Burton; G.r. W \& Engelkirk P.G. Microbiology: para las Ciencias de la salud, ed 7. Rio de Janeiro, Guanabara Koogam, 2005.

3. Vieira, f el.; Agostini, J.S. prevalencia y Perfil de infecciones causadas por Staphylococcus Aureus, aislado en un Hospital público de Dourados-MS, 2011.

4. Clínica de Spicer W.J. bacteriología, Micología y Paracitologia. Rio de Janeiro, Guanabara Koogam, 2002.

5. W Bauer; Perry D. M; Kirby W.M.M. droga uso y antibiótico susceptibilidad de estafilococos. Revista de la Asociación Médica Americana 173:475-480, 1960.

6. Cruvinel, A. R; Sen, a. R, Smith, J.S. antimicrobiano Perfil de Staphylococcus aureus aisladas de pacientes hospitalizados en terapia intensiva en el Distrito 
Federal. Cenarium Pharmacêutico, año 4, № 4, Maiol Nov de 2011.issn19843380 .

7. Moura, P. J.; Pimienta, F. C; Elía, m. et al. La colonización de staphylococcus Aureus de enfermería profesionales de la Revista Latinoamericana de enfermería, 19 (2): mar-abr 2011.

8. Castro, F. L; Barth Jr, V.C. Gallo, determinación de otros S.W.et de resistencia a los antimicrobianos de aislamientos de staphylococcus aureus. X salón de iniciación científica-PUCRS.2009.

9. Souza, L.B. G; Figueiredo, B.B. prevalencia de infecciones nosocomiales causadas por Staphylococcus Aureus resistente a meticilina (M.R.S. A), en el Hospital Universitario Regional de Maringá. RBAC, vol. 40 (1): 31-34.2008

10.Gelatti, L. C. Sukiiennik, T.et al. Sepsis por resistentes a la meticilina Staphylococcus Aureus adquirido en comunidad de la sociedad brasileña de Brasil Sur 42 revista de Medicina Tropical (4): 458-460, jul-ago.2009.

11. La revista dirigida a profesionales de salud "Hospital en foco". Año I-número 03 de septiembre de 2009.

12. Tavares, w. bacterias Gram-positivas: Staphylococcus, resistencia del Enterococo y antimicrobiana neumococo. revista de la sociedad brasileña de Medicina Tropical.33 (3): 281-301, mayo-jun.2000.

13. Ueno, M.; Jorge, A.O.C. Caracterización de Staphylococcus Aureus resistente a meticilina, involucrado en las infecciones nosocomiales, a través de técnicas fenotípicas y análisis de perfil Plasmidial. revista Bioscience., vol. 7, núm. 2, julio-diciembre de 2001.

14. ¿Wannmacher, el uso indiscriminado de antibióticos y resistencia microbiana I.: una guerra perdida? ISS 1810-0791, Vol1, $\mathrm{n} \cong$ 4, Brasilia, marzo de 2004.

15. Cruz, E.D.; Pimienta, F. C; HayashidaM. et al. Detección de Staphylococcus aureus en la boca de los trabajadores de limpieza hospitalaria. Revista-Am, enfermería, janfev.2001.w.w.w.eerp.usp.br/rlae

16. León, N.Q.et al. Enfermedades infecciosas y parasitarias: enfoque de Amazon. Editor Cejup; UEPA. Instituto Evandro Chagas, Belén, 1997. 